\title{
BMJ Open Cost-effectiveness analysis of routine pneumococcal vaccination in the UK: a comparison of the PHiD-CV vaccine and the PCV-13 vaccine using a Markov model
}

\author{
Emmanuelle Delgleize, ${ }^{1}$ Oscar Leeuwenkamp, ${ }^{2}$ Eleni Theodorou, ${ }^{3}$ \\ Nicolas Van de Velde ${ }^{1}$
}

To cite: Delgleize $\mathrm{E}$ Leeuwenkamp 0 , Theodorou $\mathrm{E}$, et al. Costeffectiveness analysis of routine pneumococcal vaccination in the UK: a comparison of the PHiD-CV vaccine and the PCV-13 vaccine using a Markov model. BMJ Open 2016;6: e010776. doi:10.1136/ bmjopen-2015-010776

- Prepublication history and additional material is available. To view please visit the journal (http://dx.doi.org/ 10.1136/bmjopen-2015010776).

Received 4 December 2015 Revised 19 August 2016 Accepted 9 September 2016

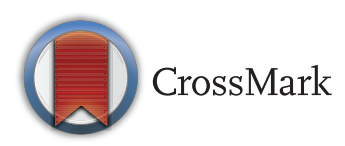

${ }^{1}$ GSK Vaccines, Wavre, Belgium

${ }^{2}$ Eclipse, Tervuren, Belgium ${ }^{3}$ GSK Pharma, London, UK

Correspondence to Dr Nicolas Van de Velde; nicolas.x.van-de-velde@gsk. com

\section{ABSTRACT}

Objectives: In 2010, the 13-valent pneumococcal conjugate vaccine (PCV-13) replaced the 7-valent vaccine (introduced in 2006) for vaccination against invasive pneumococcal diseases (IPDs), pneumonia and acute otitis media (AOM) in the UK. Using recent evidence on the impact of PCVs and epidemiological changes in the UK, we performed a cost-effectiveness analysis (CEA) to compare the pneumococcal non-typeable Haemophilus influenzae protein D conjugate vaccine (PHiD-CV) with PCV-13 in the ongoing national vaccination programme.

Design: CEA was based on a published Markov model. The base-case scenario accounted only for direct medical costs. Work days lost were considered in alternative scenarios.

Setting: Calculations were based on serotype and disease-specific vaccine efficacies, serotype distributions and UK incidence rates and medical costs.

Population: Health benefits and costs related to IPD, pneumonia and AOM were accumulated over the lifetime of a UK birth cohort.

Interventions: Vaccination of infants at 2, 4 and 12 months with PHiD-CV or PCV-13, assuming complete coverage and adherence.

Outcome measures: The incremental cost-effectiveness ratio (ICER) was computed by dividing the difference in costs between the programmes by the difference in quality-adjusted lifeyears (QALY).

Results: Under our model assumptions, both vaccines had a similar impact on IPD and pneumonia, but PHiD-CV generated a greater reduction in AOM cases (161 918), AOM-related general practitioner consultations (31 070) and tympanostomy tube placements (2399). At price parity, PHiD-CV vaccination was dominant over PCV-13, saving 734 QALYS as well as $£ 3.68$ million to the National Health Service (NHS). At the lower list price of PHiD-CV, the cost-savings would increase to $£ 45.77$ million.

Conclusions: This model projected that PHiD-CV would provide both incremental health benefits and cost-savings compared with PCV-13 at price parity.

\section{Strengths and limitations of this study}

- The study incorporates the most recent evidence on pneumococcal conjugate vaccine (PCV) efficacy/effectiveness as well as the latest epidemiological data from the UK (2014). Moreover, model parameters have been validated by a panel of external experts.

- The model is based on a previously published PCV cohort model, and an extensive sensitivity analysis has been performed to reflect uncertainty in model assumptions.

- Results are timely and relevant for policy. Current decisions did not anticipate such a large serotype replacement in the elderly, which may warrant a re-evaluation of the budget allocated to PCV based on the health benefits actually generated by the current programme.

- Static cohort models integrate net herd protection (ie, herd protection and type replacement combined) using a fixed effect at equilibrium. However, dynamic models may be more appropriate to capture these effects over time and in relation to population characteristics and vaccine coverage.

- Owing to the lack of head-to-head studies comparing the two vaccines, some efficacy estimates are from different studies and might not be directly comparable. However, model assumptions are conservative and a panel of independent experts reviewed all available evidence to select the most comparable efficacy estimates for both vaccines.

Using PHiD-CV could result in substantial budget savings to the NHS. These savings could be used to implement other life-saving interventions.

\section{INTRODUCTION}

Streptococcus pneumoniae $(S p)$ infection is established as a cause of significant morbidity and mortality in infants and young children 
worldwide. ${ }^{1}$ The WHO estimated that in $20085 \%$ of allcause mortality in children $<5$ years old was attributable to pneumococcal infections worldwide. ${ }^{2}$ Invasive pneumococcal disease (IPD), mainly meningitis and bacteraemia, is a clinical manifestation of infection with $S p$. The pneumococcus also plays a significant role in causing non-invasive infections such as pneumonia and acute otitis media (AOM). ${ }^{1}$

In the UK, 5000-6000 cases of IPD were reported annually to Public Heath England from laboratories in England and Wales before the introduction of routine childhood immunisation with pneumococcal conjugate vaccine $(\mathrm{PCV})$. In addition, there were an estimated 40000 hospitalisations due to pneumococcal pneumonia, 40000 general practitioner (GP) consultations for pneumococcal-related community-acquired pneumonia (CAP) and over 63000 GP consultations for pneumococcal otitis media $(\mathrm{OM})$ in England and Wales each year. $^{3}$

The 7-valent PCV (PCV-7) has reduced the incidence of IPD in the UK since its introduction in $2006 .{ }^{4}$ However, alongside this reduction in IPD burden, antibiotic resistance and a shift in serotype distribution involving $S p$ serotypes not covered by the vaccine have been observed. ${ }^{5-7}$ This shift in serotype distribution often leads to replacement in carriage and disease, with the potential of extension to other pathogens. It has even been shown that vaccination with PCV-7 may lead to a rise in non-typeable Haemophilus influenzae (NTHi)-related AOM. ${ }^{8}$ Therefore, the 13-valent PCV (PCV-13, Preunar 13, Pfizer, Pearl River, New York, USA) and the 10-valent pneumococcal non-typeable Haemophilus influenzae protein $\mathrm{D}$ conjugate vaccine (PHiD-CV, Synflorix, GSK group of companies, Rixensart, Belgium) were introduced in 2009. ${ }^{9}$ PHiD-CV includes three additional $S p$ serotypes $(1,5$ and $7 \mathrm{~F})$ compared with PCV-7, while PCV-13 includes these additional serotypes and three further $S p$ serotypes (3, 6A and 19A). Although $S p$ serotypes $6 \mathrm{~A}$ and $19 \mathrm{~A}$ are not included in PHiD-CV, clinical evidence suggests that PHiD-CV offers marked protection against these cross-reactive $S p$ serotypes. ${ }^{10-15}$ So far, there is no conclusive evidence that PCV-13 prevents IPD, AOM or pneumonia due to serotype $3 .^{16}{ }^{17}$ Furthermore, in contrast to PCV-7 and PCV-13, PHiD-CV has the additional potential to target NTHi-related AOM, due to the employment of protein $\mathrm{D}$ derived from $\mathrm{NTHi}$ as a carrier protein for the majority of its conjugates. ${ }^{18} 19$ This is important, as AOM represents a major indication for the prescription of antibiotics in infants. ${ }^{20}{ }^{21}$ Significant antibiotic resistance has been observed in $S p^{22-24}$ and NTHi bacteria, ${ }^{25}$ and the increasing resistance to antibiotics is globally recognised as a health policy concern.

Infectious disease modelling integrates data from multiple sources (eg, epidemiological, economic, demographic and biological) to predict the impact of new interventions in a given population. In 2012, a Markov cohort model evaluating the cost-effectiveness of
PHiD-CV versus PCV-13 for Canada and the UK was published by Knerer et $a l,{ }^{26}$ using epidemiological information from 2006 to 2007 (ie, before the introduction of PCV-7). Since then the situation has changed, in terms of epidemiology in the UK (eg, serotype distribution and IPD incidence), and because more evidence is now available regarding the impact of both vaccines (eg, protection against cross-reactive $S p$ types $6 \mathrm{~A}$ and 19A). Here, we present an updated cost-effectiveness analysis comparing PHiD-CV with PCV-13 in the current UK setting.

\section{MATERIALS AND METHODS \\ Model overview}

The Markov model described here is conceptually identical to the model published earlier by Knerer $e t$ al and is essentially an update of that model. ${ }^{26}$ Figure 1 shows the flow diagram of the model. Model input data and assumptions were based on published data where possible, and were validated where appropriate by a panel of independent experts (GSK PHiD-CV Health Economics Advisory Board, Leuven, Belgium, September, 2013).

This model was used to estimate the epidemiological and economic impact of a universal infant pneumococcal vaccination programme in the UK. The model simulated the impact of vaccination on invasive and

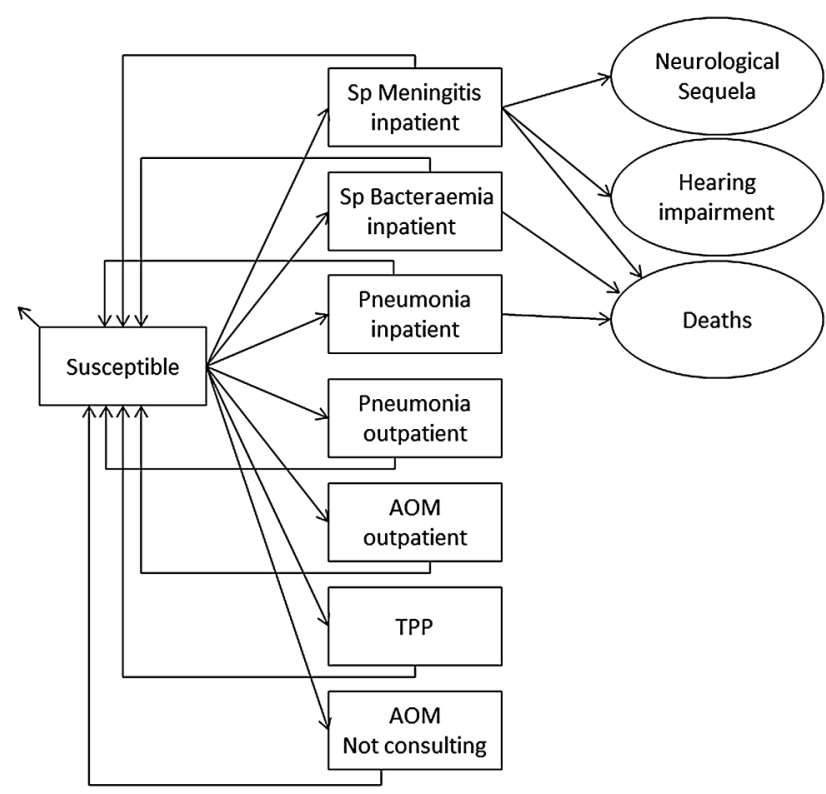

Figure 1 Model flow diagram. Rectangles represent mutually exclusive health states. Age-specific incidences are applied monthly to the susceptible population. Circles (sequelae and death) and small arrows (natural death) represent the proportion of the population removed from the model. Costs and benefits are computed monthly and aggregated over the cohort's lifetime. Non-consulting AOM episodes are accounted for in the quality of life calculation. AOM, acute otitis media; Sp, Streptococcus pneumoniae; TTP, tympanostomy tube placement. 
non-invasive diseases caused by $S p$ and NTHi-related infections in a birth cohort. It compared two PCVs, PCV-13 (currently available in the UK) and PHiD-CV, each given in a three-dose $(2+1)$ schedule with vaccine doses administered at 2, 4 and 13 months of life.

Individuals in the birth cohort were followed in the model over a lifetime with a cycle time of 1 month. During each model cycle, the probability of an individual entering a specific health state was governed by agespecific incidence rates and applicable vaccine efficacy (VE) levels. These transition probabilities determined hospitalisation rates and frequency of medical visits associated with the disease conditions considered in the model. Costs and quality-adjusted life-years (QALYs) lost associated with the model outcomes (eg, cases, medical visits, hospitalisations) were accumulated over the cohort's lifetime. The base-case analysis took the perspective of the healthcare provider in the UK, the National Health Service (NHS), and therefore accounted only for direct medical costs. An additional analysis accounting for productivity loss was conducted, providing a broader perspective that may further help inform the decision-making process.

\section{Epidemiological data \\ Demography}

The size of the birth cohort in the UK ( $\mathrm{n}=792616$ infants; reference year mid-2013) and age-specific overall monthly mortality rates for the general population were obtained from the Office for National Statistics (ONS; see online supplementary table S1 in file 1). ${ }^{27} 28$

\section{Invasive pneumococcal diseases}

This section describes the parameters used for the $S p$ meningitis and bacteraemia natural histories in figure 1 . In the model, all cases of IPD were assumed to be hospitalised. Transitions from the susceptible state to $S p$ meningitis and bacteraemia requiring inpatient admission were derived from age-specific hospital admission data from the Hospital Episode Statistics (HES) database 2013-2014, and population age distribution data from the ONS (see online supplementary table S2 in file 1). ${ }^{28} 29$ In the HES database, the International Classification of Disease V.10 (ICD-10) codes used for meningitis and bacteraemia are G00.1 (pneumococcal meningitis) in the primary diagnosis fields and A40.3 (sepsis due to $S p$ ) in all diagnosis fields, respectively. ${ }^{29}$ All diagnosis fields were used for bacteraemia as this disease condition occurs frequently as a complication. The pathogen-specific ICD-10 codes applied are in line with the definitions in studies used for VE estimates (see Vaccine efficacy section). Following meningitis, children and adults can develop long-term neurological sequelae and hearing impairment. The proportion of children with neurological sequelae $(7.0 \%)$ and hearing impairment $(13.3 \%)$ were based on Pomeroy et $a \ell^{00}$ and a meta-analysis of 11 studies, ${ }^{31}$ respectively. For adults, the proportion of meningitis cases with neurological sequelae $(19.0 \%)$ and hearing impairment $(25.4 \%)$ were based on the weighted average of two studies by Kastenbauer and Pfister ${ }^{32}$ and Auburtin et al. ${ }^{33}$ As data are limited in the UK, we conservatively assumed no long-term sequelae following an episode of bacteraemia. Similarly, cases and deaths due to NTHi ID were not included in the base-case analysis. Case-fatality ratios (CFRs) for $S p$ meningitis and bacteraemia were extracted from Johnson et $a l^{34}$ and Melegaro and Edmunds, ${ }^{35}$ respectively. For children aged $\leq 4$ years, CFR data were further updated using Ladhani et $\mathrm{al}^{7}$

\section{Pneumonia}

Inpatient incidence rates of all-cause pneumonia were derived from the HES database using ICD-10 codes J13 $(S p), \mathrm{J} 14(H i), \mathrm{J} 15.9$ (bacterial pneumonia, unspecified) and J18.1/8/9 (lobar pneumonia or unspecified (pneumonia/pathogen); see online supplementary table S3 in file 1) ${ }^{29}$ The broad set of ICD-10 codes considered is in line with efficacy end points reported in published clinical trials not discriminating between causative pathogens (see Vaccine efficacy section). Age-specific CFRs for inpatient pneumonia were based on data from Melegaro and Edmunds. ${ }^{35}$ Outpatient incidence rates were based on 2010 data from the Royal College of General Practitioners (RCGP) in England and Wales ${ }^{36}$ and no mortality was assumed.

\section{Acute otitis media}

Outpatient incidence rates for AOM episodes (not visits) were taken from the RCGP 2011 Annual Report (ICD-9 codes 381.0-acute non-suppurative OM, 382.0-acute suppurative $\mathrm{OM}$ and 382.9 -unspecified $\mathrm{OM}) .{ }^{36}$ The proportion of AOM cases caused by $S p(35.9 \%)$, NTHi $(32.3 \%)$ and other causative agents $(31.8 \%)$ were extracted from the review of Leibovitz et al. ${ }^{37}$ AOM cases caused by $S p$ were further distributed between pneumococcal serotypes based on a multinational meta-analysis of nine data sets published by Hausdorff $e t a l^{38}$ The agespecific rate of tympanostomy tube placement (TTP) was obtained from the HES database using the procedural code D15.1 (myringotomy with insertion of ventilation tube through the tympanic membrane; see online supplementary table $\mathrm{S} 4$ in file 1). ${ }^{39}$ The model accounted for the reduced quality of life of patients with AOM not consulting a GP using an adjustment factor, defined as the ratio of GP consultations from Williamson et $a t^{40}$ over the total number of $\mathrm{AOM}$ cases from Melegaro and Edmunds. ${ }^{35}$ Finally, our model assumed no complications, long-term sequelae or deaths related to AOM.

\section{Costs}

All costs were reported in British pounds sterling (£) with 2014 as the reference year. Costs prior to 2014 were adjusted on the basis of the UK healthcare service cost index (V.02 May 2013). ${ }^{41}$ The direct annual costs per 
acute episode are given in the online supplementary table S1 in file 2. NHS reference costs (2013-2014) were used to identify cost components including those dependent on disease conditions: for example, ambulance transfer, accident and emergency investigation, intensive care unit stay, CT/MRI scanning or X-ray, ultrasound, weighted average cost of hospital stay and cost of primary care consultation, using information from Melegaro and Edmunds ${ }^{35}$ where applicable. Costs associated with meningitis consisted of two components, the costs of treating the initial meningitis episode and the follow-up costs associated with long-term sequelae (neurological sequelae and hearing loss) incurred over the remaining lifetime. The incidences of sequelae for bacteraemia, all-cause pneumonia and AOM were conservatively set to zero in the model. In these instances, disease-related treatment costs were assumed to be incurred within 1 month with no long-term costs due to sequelae.

In the base-case scenario, price parity was assumed for PHiD-CV and PCV-13. Total vaccine costs per dose were estimated from the list price, accounting for $5 \%$ wastage and an administration cost of $£ 7.64$ per dose. ${ }^{42}$ The resultant total vaccine cost per dose was estimated at $£ 48.88$ based on a $0.5 \mathrm{~mL}$ prefilled syringe. Vaccination coverage was assumed to be $100 \%$, in line with vaccination rates commonly obtained with national immunisation programmes in the UK. ${ }^{43}$ Furthermore, a complete course (three doses) with perfect adherence (100\%) was assumed. Although these assumptions on coverage and adherence are simplified, they affect both vaccines equally and therefore should not have an impact on the cost-effectiveness ratio.

\section{Utilities}

Three types of utility values were used in the model. Normative utility values represent the age-specific utility in healthy individuals. ${ }^{44}$ The QALY loss per episode was computed for acute diseases using the formula (1 -QALY weight $) \times($ duration of episode in days $/ 365$ days $)$. The QALY loss per year (1-QALY weight) was used for long-term conditions (see online supplementary table S1 in file 3)..$^{35-49}$ In the literature, studies looking at long-term meningitis sequelae have variable follow-up times (5-20 years).$^{50}$ In our model and others, disutility weights for long-term sequelae were incurred for the time remaining until the end of the study time horizon.

\section{Vaccine efficacy}

In the base-case analysis, the impact of vaccination was assessed including both direct and indirect effects of protection (herd protection). To estimate the direct effect of vaccination, published estimates of $\mathrm{VE}$ were applied to the age-specific disease incidence in sequential model cycles. The VE estimates used in the model and described in this section have been validated by a panel of independent experts (GSK PHiD-CV Health
Economics Advisory Board, Leuven, Belgium, September, 2013; table 1).

\section{Invasive pneumococcal diseases}

Overall efficacy against meningitis and bacteraemia was computed for both vaccines based on the serotype-specific efficacy estimates from Whitney et $a l^{13}$ and the local serotype distribution derived from Waight et al (see online supplementary table S5a in file 1). ${ }^{51} 52$ The efficacy used for all vaccine serotypes included in PHiD-CV and PCV-13 was the average efficacy reported by Whitney et al against PCV-7 serotypes (serotype-specific efficacy estimates were not used as for some serotypes the number of cases was too small) ${ }^{13}$ except for serotype 3 . Serotype 3 is included in PCV-13 but not in PHiD-CV. However, both PCV-13 and a precursor of PHiD-CV have failed to show significant efficacy against serotype $3,{ }^{1016175153-56}$ probably because of its thicker polysaccharide capsule.

In addition to efficacy against vaccine serotypes, large PCV clinical trials have shown that serotypes $19 \mathrm{~F}$ and $6 \mathrm{~B}$ can also induce protection against $19 \mathrm{~A}$ and $6 \mathrm{~A}$, respectively, because they functionally belong to the same serogroup. $^{11}{ }^{13-15}$ Cross-protection against serotype 6A (76\%) owing to serotype 6B (included in all PCVs) has been demonstrated in PCV-7 trials. ${ }^{13} 57$ Finally, large clinical trials in Brazil, ${ }^{11}$ Canada $^{14}$ and Finland ${ }^{15}$ have reported VE against 19A in IPD of $82 \%, 71 \%$ and $62 \%$, respectively, which prompted an update of the Summary of Product Characteristics (SmPC) for PHiD-CV to include protection against 19A (the European Medicines Agency (EMA) approval on 23 July 2015) ${ }^{58}{ }^{59}$ In this analysis, we used the Finnish estimate for VE $(62 \%)$ because it is based on a $2+1$ PHiD-CV vaccination schedule administered in a European setting. ${ }^{15}$

\section{Pneumonia}

VE of PCVs against CAP has been assessed in several large-scale, randomised, controlled trials conducted in different settings. ${ }^{12}{ }^{60-64}$ These trials have shown that protection against disease is not associated with the number of serotypes included in the vaccine formulation. Hence, the model assumed an efficacy of $23.4 \%$ against X-ray-confirmed consolidated CAP (which usually requires hospitalisation) and $7.3 \%$ against clinically suspected CAP (commonly managed on an outpatient basis) for both vaccines, based on a clinical trial of PHiD-CV. ${ }^{12}$ These estimates are conservative, as postmarketing surveillance data from Brazil have shown a reduction of $40 \%$ and $30 \%$ in pneumonia hospitalisations with PHiD-CV ${ }^{65}$ and PCV-13, ${ }^{66}$ respectively.

\section{Acute otitis media}

VE against all vaccine types except serotype 3 (same as for IPD $^{16}$ ) was assumed to be $69.9 \%$ for both vaccines. ${ }^{12}$ VE against non-vaccine types was assumed to be $-33.0 \%$ for both vaccines to account for serotype replacement. ${ }^{67}$ PHiD-CV cross-protection against serotype 6A (63.7\%) was based on Prymula et $a l^{10} 12$ and the cross-protection 
Table 1 Model input data: VE

\begin{tabular}{|c|c|c|c|}
\hline & \multicolumn{2}{|l|}{ VE* \% $(95 \% \mathrm{Cl})$} & \multirow[b]{2}{*}{ Reference/assumption } \\
\hline & PHiD-CV & PCV-13 & \\
\hline \multicolumn{4}{|l|}{ IPD } \\
\hline Ten common serotypes for PHiD-CV and PCV-13† & $\begin{array}{l}94.7 \ddagger \\
\text { (87.0 to100.0) }\end{array}$ & $\begin{array}{l}94.7 \ddagger \\
\text { (87.0 to100.0) }\end{array}$ & Whitney et $a l^{13}$ \\
\hline Serotype 3 & 0.0 & $\begin{array}{l}0.0 \\
(26 \% \text { in } S A)\end{array}$ & Andrews et $a l^{17}$ \\
\hline Serotype $6 \mathrm{~A}$ & $\begin{array}{l}76.0 \\
(39.0 \text { to } 90.0)\end{array}$ & $\begin{array}{l}94.7 \\
(87.0 \text { to } 100.0)\end{array}$ & $\begin{array}{l}\text { Vesikari et } a{ }^{56} \\
\text { Whitney et } a l^{13}\end{array}$ \\
\hline Serotype $19 A$ & $\begin{array}{l}62.0 \\
(20 \text { to } 85)\end{array}$ & $\begin{array}{l}94.7 \\
(87.0 \text { to } 100.0)\end{array}$ & $\begin{array}{l}\text { Whitney et al } \\
\text { Jokinen et } a l^{15}\end{array}$ \\
\hline \multicolumn{4}{|l|}{ Pneumonia } \\
\hline Per cent of reduction in hospitalisations & $\begin{array}{l}23.4 \\
\text { (8.8 to } 35.7)\end{array}$ & $\begin{array}{l}23.4 \\
\text { (8.8 to } 35.7)\end{array}$ & Tregnaghi et al ${ }^{12}$ \\
\hline Per cent of reduction in GP visits & $\begin{array}{l}7.3 \\
\text { (2.1 to } 12.3)\end{array}$ & $\begin{array}{l}7.3 \\
\text { (2.1 to } 12.3)\end{array}$ & \\
\hline \multicolumn{4}{|l|}{ AOM without TTP } \\
\hline Ten common serotypes for PHiD-CV and PCV-13 & $\begin{array}{l}69.9 \\
(29.8 \text { to } 87.1)\end{array}$ & $\begin{array}{l}69.9 \\
(29.8 \text { to } 87.1)\end{array}$ & Tregnaghi et al ${ }^{12}$ \\
\hline Non-vaccine type Streptococcus pneumoniae & $\begin{array}{l}-33.0 \\
(-33.0 \text { to } 15.0)\end{array}$ & $\begin{array}{l}-33.0 \\
(-33.0 \text { to } 15.0)\end{array}$ & Eskola et $a{ }^{67}$ \\
\hline Serotype 3 & 0.0 & 0.0 & As for IPD \\
\hline Serotype $6 A$ & $\begin{array}{l}63.7 \\
(-13.9 \text { to } 88.4)\end{array}$ & $\begin{array}{l}69.9 \\
(29.8 \text { to } 87.1)\end{array}$ & $\begin{array}{l}\text { Prymula et al }{ }^{10} \\
\text { Tregnaghi et al }\end{array}$ \\
\hline Serotype 6C & 0.0 & $\begin{array}{l}63.7 \\
(-13.9 \text { to } 88.4)\end{array}$ & Same as $6 \mathrm{~A}$ for PHiD-CV \\
\hline Serotype 19A & $\begin{array}{l}45.8 \S \\
(-33 \text { to } 15)\end{array}$ & $\begin{array}{l}69.9 \\
(29.8 \text { to } 87.1)\end{array}$ & Tregnaghi et al ${ }^{12}$ \\
\hline $\mathrm{NTHi}$ & $\begin{array}{l}21.5 \\
(-43.4 \text { to } 57.0)\end{array}$ & $\begin{array}{l}-11.0 \\
(-34 \text { to } 8)\end{array}$ & $\begin{array}{l}\text { Tregnaghi et al }{ }^{12} \\
\text { Eskola et } a f^{7}\end{array}$ \\
\hline \multicolumn{4}{|l|}{ AOM with TTP } \\
\hline TTP & 50.9 & 30.6 & $\begin{array}{l}\text { Extrapolated }\rceil \text { from } \\
\text { Fireman et a }{ }^{68} \\
\text { and Palmu et } a{ }^{69}\end{array}$ \\
\hline
\end{tabular}

${ }^{*}$ All VE estimates have been validated by an expert panel.

tIncluded serotypes were 1, 4, 5, 6B, 7F, 9V, 14, 18C, 19F and 23F.

$\ddagger$ PHiD-CV and PCV-13 were assumed to have the same serotype efficacy for the 10 common serotypes as the average VE of PCV-7 vaccine serotypes $(94.7 \%)$.

$\S P H i D-C V$ efficacy was estimated taking the efficacy ratio of the vaccines in IPD (vaccine serotypes).

TExtrapolated VE estimates were well in agreement with findings of the FinIP study: ${ }^{70}$ the boundaries reflect $95 \%$ Cls that were used in the sensitivity analyses.

AOM, acute otitis media; FinIP, Finnish Invasive pneumococcal disease; GP, general practitioner; IPD, invasive pneumococcal disease; JCVI, Joint Committee on Vaccination and Immunisation; PCV-13, 13-valent pneumococcal conjugate vaccine; PHiD-CV, 10-valent pneumococcal non-typeable Haemophilus influenzae protein D conjugate vaccine; SA, sensitivity analysis; Sp, Streptococcus pneumoniae; TTP, tympanostomy tube placement; VE, vaccine efficacy.

against $19 \mathrm{~A}(45.7 \%)$ was computed using the ratio of efficacy estimates between PHiD-CV and PCV-13 in conjunction for IPD. VE against NTHi-related AOM was assumed to be $21.5 \%$ for PHiD-CV. ${ }^{10} 1267$ For PCV-13, the $\mathrm{VE}$ was $-11.0 \%$, as reported for its predecessor PCV-7. ${ }^{67}$ These efficacy estimates, in line with expert validation and with data reported by Tregnaghi et al, ${ }^{12}$ were applied to the respective serotypes, taking into account the frequency of these serotypes in causing AOM (see online supplementary table S5b in file 1) ${ }^{66}{ }^{67}$ A similar approach was used for NTHi-related AOM. VE for both vaccines against AOM with TTP procedures was extrapolated using an exponential function based on the results of PCV-7 clinical trials ${ }^{68} 69$ and the relative ratio of overall AOM VE. ${ }^{70}$ These VE estimates were in agreement with the findings of the Finnish Invasive pneumococcal disease (FinIP) study. ${ }^{70}$ The higher efficacy of PHiD-CV compared with PCV-13 for AOM is reflected in the estimates of VE against TTP procedures (table 1).

Direct vaccine protection in children against IPD, pneumonia and AOM is age-specific. First, during the ramp-up phase VE increases to $50 \%, 90 \%$ and $100 \%$ of the type-specific and disease-specific VE estimates described above following the first, second and booster doses, respectively. ${ }^{12}{ }^{68}$ Second, from 13 months (booster dose) to 3 years of age we assumed that $\mathrm{VE}$ does not wane. ${ }^{12}$ Finally, VE was assumed to wane exponentially from 3 to 10 years of age, after which the 
vaccine stops providing any direct protection to the vaccinated birth cohort. ${ }^{71}$ These assumptions are conservative, since data are now available showing that efficacy does not decrease exponentially after 3 years. ${ }^{12}$

Indirect or herd protection resulting from continual vaccination of sequential birth cohorts was taken into account for the entire population for IPD only (we conservatively assumed no herd protection for pneumonia and AOM). Serotype replacement offsets the incremental effect of indirect protection. In the model, indirect protection adjusted for the opposing impact of serotype replacement was applied as a fixed effect to the residual disease incidence. This net indirect effect was estimated at $30 \%$, removing the necessity to account separately for the effect of serotype replacement. ${ }^{72} 73$ All efficacy estimates and the net indirect effect applied in the model are in line with Tregnaghi $e t a l^{12}$ and were validated by a panel of experts (GSK PHiD-CV Health Economics Advisory Board, Leuven, Belgium, September, 2013). Assumptions regarding net herd protection were varied in scenario analyses $(15 \%$ and $0 \%)$, as recent data from the UK suggest high levels of serotype replacement in adults. ${ }^{51} 7475$

\section{Cost-effectiveness and sensitivity analysis}

Health benefits and costs were accumulated over the cohort's lifetime and discounted at a rate of $3.5 \%$ to estimate the incremental cost-effectiveness ratio (ICER) ${ }^{76}$ The ICER of PHiD-CV compared with PCV-13 (expressed in $£ /$ QALY) was computed by dividing the difference in costs between the two vaccines by the difference in health outcomes.

Sensitivity analyses were performed to capture the effects of parameter uncertainty on model predictions and identify the most influential parameters. One-way sensitivity analyses were performed by varying each parameter one by one within a range of plausible estimates and ranking the parameters based on their impact on the results. In addition, a probabilistic sensitivity analysis (PSA) was performed by simultaneously varying all parameters to capture their conjoint uncertainty (500 different parameter sets sampled from probability distributions). Results from the PSA were plotted in a cost-effectiveness plane (QALYs vs costs), along with the base-case ICER estimate. Ranges used in the one-way sensitivity analyses and the probability distributions used in the PSA are provided in the online supplementary table S1 in file 4.

In addition to sensitivity analyses, we performed scenario analyses on specific model assumptions to better understand their impact on the results. Alternative scenarios explored were as follows: (1) net herd protection reduced to $15 \%$ and $0 \%$ to explore the impact of the increased serotype replacement observed in recent years in adults in the UK. ${ }^{51} 7475$ (2) Efficacy against serotype 3 increased to $26 \%$ for PCV-13 (non-significant result from $^{17}$ ). (3) NTHi ID (meningitis and bacteraemia) and NTHi pneumonia included in the model; in this scenario, the incidence of NTHi ID was assumed to be $5 \%$ of all $S p$ IPD cases in children aged $<10$ years, with a CFR of $10 \%$. (4) Productivity loss, in which in addition to direct medical costs the model also estimated the time lost from work by patients of working age (18-75 years) or time lost from work by parents caring for their sick children. For patients, the time loss estimates were multiplied by the estimated annual earnings at the individual's age, and for working parents (aged 18-49 years) the time estimates were multiplied by an average annual earnings of $£ 20375$ (see online supplementary tables S2 and S3 in file 2). ${ }^{77} 78$ (5) Accounting for the difference in list price of both vaccines in the UK $(£ 49.10 /$ dose and $£ 27.60 /$ dose for PCV-13 and PHiD-CV, respectively). ${ }^{79}$

\section{RESULTS}

\section{Base-case analysis}

Under our model assumptions, PCV-13 and PHiD-CV showed identical reductions in mortality due to IPD and all-cause pneumonia. The estimated impact on the number of IPD and pneumonia cases was also similar for both vaccines. However, PHiD-CV would prevent an additional 31070 GP visits and 2399 AOM-related TTP procedures, compared with PCV-13 (table 2).

Assuming price parity for both vaccines, PHiD-CV would reduce QALY loss by 734 QALYs (812 QALYs undiscounted) compared with PCV-13 and save $£ 3.68$ million (£4.14 million undiscounted) in direct medical costs to the NHS (dominant intervention). These results are due to fewer AOM-related GP consultations and in-hospital TTP procedures with PHiD-CV than PCV-13. For instance, the reduction in AOM-related GP visits alone would save $£ 1.43$ million to the healthcare system (table 2).

\section{Sensitivity analyses}

One-way sensitivity analyses showed that the model outcome (PHiD-CV dominated PCV-13) was robust to variations in model assumptions. Variables related to AOM (eg, disutility during an episode of AOM in outpatients, cost and reduction in TTP, AOM-related GP visits) were identified as key drivers of the cost-effectiveness results (see figure 2). The PSA showed that the model results were also robust to simultaneous variation of the parameters. The cost-acceptability curve estimated that PHiD-CV was more cost-effective compared with PCV-13 in $88 \%$ of simulations, with a cost-effectiveness threshold of £20 000/QALY (figure 3).

\section{Alternative scenarios}

Table 3 reports the difference in total QALYs gained (discounted) and total costs (discounted) between the vaccines for a range of alternative scenarios. All scenarios showed more health benefits and cost-savings for PHiD-CV compared with PCV-13. Scenario 1, reducing net herd protection to $15 \%$ and $0 \%$, did not affect the base-case results because both vaccines are affected equally. Scenario 2, accounting for the non-significant 
Table 2 Model outcomes for the base-case analysis

\begin{tabular}{|c|c|c|c|}
\hline & PHiD-CV & PCV-13 & $\begin{array}{l}\text { Incremental } \\
\text { (PHiD-CV vs PCV-13) }\end{array}$ \\
\hline \multicolumn{4}{|l|}{ Health outcomes, undiscounted (n) } \\
\hline Pneumococcal meningitis & 266 & 265 & +1 \\
\hline Pneumococcal bacteraemia & 720 & 719 & +1 \\
\hline All-cause pneumonia* & 356292 & 356292 & 0 \\
\hline $\mathrm{AOM} \dagger$ related GP visits & 933162 & 964232 & -31070 \\
\hline AOM with TTP & 29585 & 31984 & -2399 \\
\hline Cases of meningitis sequelae & 86 & 86 & 0 \\
\hline Deaths (meningitis, bacteraemia, pneumonia) & 90437 & 90437 & 0 \\
\hline Total QALYs gained, undiscounted & 53962843 & 53962031 & 812 \\
\hline Total QALYs gained, discounted & 18981547 & 18980813 & 734 \\
\hline \multicolumn{4}{|l|}{ Economic outcomes (£k), undiscounted } \\
\hline Vaccination including administration & 115997 & 115997 & 0 \\
\hline Pneumococcal meningitis & 1953 & 1948 & 5 \\
\hline Meningitis sequelae & 4592 & 4579 & 12 \\
\hline Pneumococcal bacteraemia & 4548 & 4542 & 6 \\
\hline All-cause pneumonia* & 1271214 & 1271215 & -1 \\
\hline AOM related GP visits & 42925 & 44355 & -1429 \\
\hline AOM with TTP & 33777 & 36516 & -2739 \\
\hline Total direct costs (£k), undiscounted & 1475006 & 1470151 & -4145 \\
\hline Total direct costs (£k), discounted & 297248 & 300930 & -3682 \\
\hline ICER, undiscounted & Dominant & & \\
\hline ICER, discounted & Dominantł & & \\
\hline \multicolumn{4}{|c|}{$\begin{array}{l}\text { *All-cause pneumonia includes patients hospitalised or only visiting the GP. } \\
\text { †Total AOM cases (GP visits+TTP+not consulting GP) for PHiD-CV (3 882 967) and PCV-13 (4 044 886) based on AOM adjustment factor } \\
\text { (online supplementary table S4 in file 1). } \\
\text { fPHiD-CV dominant because it provides both lower costs and higher effectiveness (larger number of QALYs) compared with PCV-13. } \\
\text { AOM, acute otitis media; GP, general practitioner; ICER, incremental cost-effectiveness ratio; PCV-13, 13-valent pneumococcal conjugate } \\
\text { vaccine; PHiD-CV, 10-valent pneumococcal non-typeable Haemophilus influenzae protein D conjugate vaccine; QALY, quality-adjusted } \\
\text { life-year; TTP, tympanostomy tube placement. }\end{array}$} \\
\hline
\end{tabular}

efficacy of $26 \%$ against serotype 3 for PCV-13, had only a small impact on the results (731 QALYs gained and £3.67 million saved with PHiD-CV vs PCV-13). Scenario 3, including NTHi ID/pneumonia (non-significant), would further increase the projected health benefits and associated savings provided by PHiD-CV (739 QALYs gained and £3.82 million saved with PHiD-CV vs PGV-13). Scenario 4, accounting for work days lost in addition to direct medical costs, increased the cost-savings to $£ 5.13$ million. Finally, Scenario 5 showed that accounting for the difference in list price between the two vaccines would result in cost-savings of $£ 45.77$ million.

\section{DISCUSSION}

We evaluated the effectiveness and cost-effectiveness of PHiD-CV vaccination in children compared with PCV-13 in the UK setting by updating a previously published Markov model. ${ }^{26}$ The present analysis showed a similar impact for both vaccines on the incidence of meningitis, bacteraemia and pneumonia. The main reasons for this finding include a low incidence of IPD, and new evidence indicating a lack of significant protection against serotype 3 for $\mathrm{PCV}-13^{17}$ and marked cross-protection provided by PHiD-CV against serotypes $6 \mathrm{~A}$ and $19 \mathrm{~A} .{ }^{18}$ However, our model predicted substantially fewer cases of AOM for PHiD-CV compared with PCV-13. This would translate into considerable cost-savings and health benefits because of the high incidence of AOM. PHiD-CV was consistently shown to be dominant over PCV-13 (ie, PHiD-CV would provide more health benefits at a lower cost) in the sensitivity analyses (one-way and PSA) and alternative scenarios. The one-way sensitivity analysis also indicated that AOM-related model parameters were the primary drivers of cost-effectiveness results.

From an epidemiological perspective, declining IPD incidence may suggest that more emphasis should be placed on the control of AOM.

AOM constitutes the prime indication for antibiotic prescription in infants, ${ }^{20} 21$ hence pneumococcal vaccination by reducing the number of AOM cases could play a role in limiting the development of antibiotic resistance. In a cluster-randomised, double-blind trial, Palmu et $a l^{80}$ reported that the use of $\mathrm{PHiD}-\mathrm{CV}$ vaccine in Finland could result in yearly 12000 fewer outpatient antibiotic purchases for AOM in children aged $<2$ years. Assuming similar treatment practices in Finland and the UK, and accounting for the difference in number of AOM cases prevented between PHiD-CV and PCV-13 (161 918 AOM cases prevented) or no vaccination (423 339 AOM cases prevented), the extrapolation 


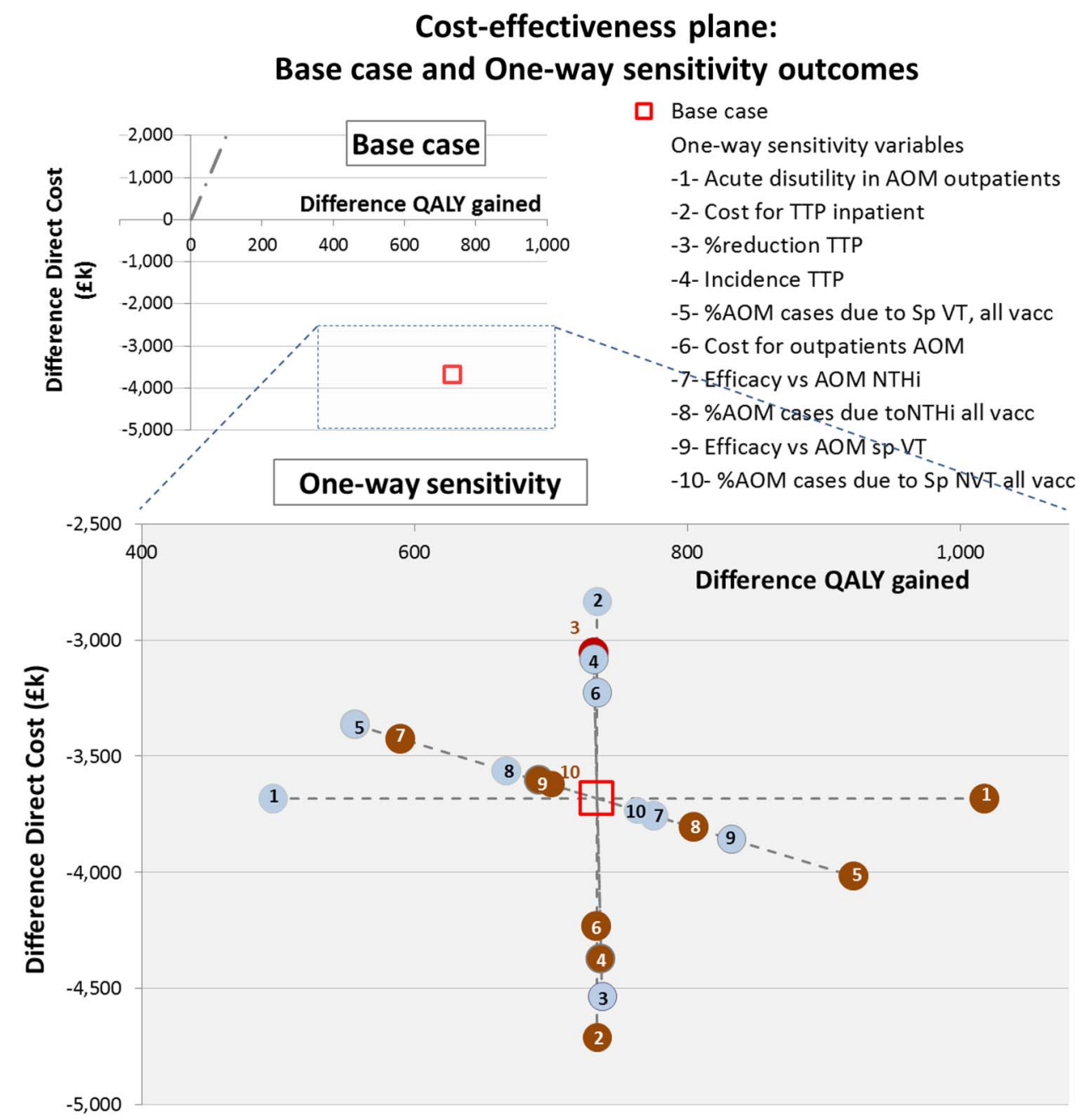

Figure 2 One-way sensitivity analysis. Effect on the difference in QALY gained and on the difference in direct cost using the lower (light blue bullet) and upper bound value (brown bullet) of one parameter (-n-) and keeping all other parameters equal to the estimated value that is used for calculating the base case. Each parameter will provide two ICERs according its lower and upper bound (values in online supplementary table $\mathrm{S} 1$ in file 4). Parameter are ranked in descending order according to the spread between their two ICERs, with the highest spread referring to the most influencing parameter in the CEA analysis (acute disutility for AOM outpatient). AOM, acute otitis media; CEA, cost-effectiveness analysis; comparator=PHiD-CV, pneumococcal non-typeable Haemophilus influenzae protein D conjugate vaccine; GP, general practitioner; ICER, incremental cost-effectiveness ratio (=difference in direct cost divided by difference in QALY gained divided); NTHi, non-typeable Haemophilus influenzae; QALY, quality-adjusted life-year; Sp, Streptococcus pneumonia; standard intervention=PCV-13, 13-valent pneumococcal conjugate vaccine; VT, vaccine type.

of the Finish results to the UK settings would translate into about 60700 fewer antibiotic purchases per year in children aged $<2$ years between PHiD-CV and PCV-13. Conceptually, this updated model is identical to the previously published model evaluating the economic impact of PHiD-CV. ${ }^{26}$ The present model used updated parameters including recent epidemiological information (age-specific hospitalisation and GP consultation rates due to $S p$ meningitis, $S p$ bacteraemia, pneumonia and AOM including the incidence of inpatient TTP), recent VE data, cross-protection and evidence-based approximations of indirect protection. Furthermore, age-stratified serotype distribution for IPD could be constructed for 2013-2014 based on the recent data reported by Waight et al. ${ }^{51}$ Costs were indexed to 2014 values as appropriate and productivity loss was included in the scenario analysis. The baseline year of the updated model was 2013-2014, and accommodated the decline in the incidence of IPD seen in the UK since the introduction of PCV-7 in 2006 and subsequent implementation of PCV-13 in 2010. Against this epidemiological background, re-evaluation of the influence of vaccination strategies on disease incidence, costs, health gain and ICER is considered of interest.

While the cost-effectiveness profiles of different pneumococcal vaccination strategies in several countries have been previously evaluated using similar tools such as Markov cohort models, direct comparison of results is 


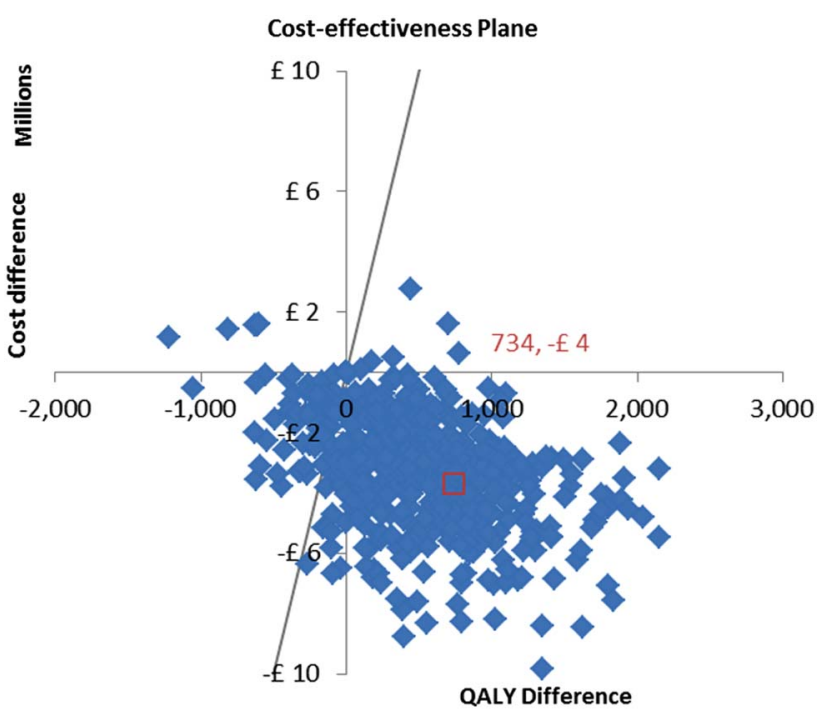

- Individual simulations $\quad \square$ Base case $\quad$ Cost-effectiveness threshold

Figure 3 Probabilistic sensitivity analysis. Probabilistic distributions have been defined around each parameter and 500 different parameter sets were sampled by varying all parameters simultaneously. The red point in the cost-effectiveness plane represents the base-case parameter set. QALY, quality-adjusted life-year.

Table 3 Model outcomes from the scenario analyses

\begin{tabular}{|c|c|c|}
\hline \multirow[b]{2}{*}{ Scenario } & \multicolumn{2}{|c|}{ PHiD-CV vs PCV-13 } \\
\hline & $\begin{array}{l}\text { Cost } \\
\text { difference* }(£)\end{array}$ & $\begin{array}{l}\text { QALY } \\
\text { difference }\end{array}$ \\
\hline Base case & -3681976 & 734 \\
\hline No net herd protection & -3681967 & 734 \\
\hline $\begin{array}{l}\text { Serotype } 3 \text { efficacy } \\
\text { against IPD }=26 \% \text { in } \\
\text { PCV-13 }\end{array}$ & -3669466 & 731 \\
\hline $\begin{array}{l}\text { NTHi ID/pneumoniat } \\
\text { included }\end{array}$ & -3816273 & 739 \\
\hline Including productivity loss & -5132994 & 734 \\
\hline Difference in list price $\neq$ & -45770435 & 734 \\
\hline \multicolumn{3}{|c|}{$\begin{array}{l}{ }^{*} \text { Cost difference estimated on the basis of the number of } \\
\text { consultations instead of episodes, using the RCGP } 2011 \text { weekly } \\
\text { report data (from RCGP in England and Wales) and identical } \\
\text { QALY gain assumed in deriving the associated ICER. } \\
\text { †Assumed incidence rate NTHi IPD } 5 \% \text { (expert opinion) and } \\
\text { efficacy as in AOM; estimated incidence NTHi pneumonia cases } \\
3 \% \text { and percentage reduction in hospitalisation in accordance with } \\
\text { efficacy in AOM. } \\
\ddagger \text { In the UK, PCV-13 list price= }=49.10 \text { and PHiD-CV list price= } \\
£ 27.60 \text {. } \\
\text { AOM, acute otitis media; ICER, incremental cost-effectiveness } \\
\text { ratio; ID, invasive disease; IPD, invasive pneumococcal disease; } \\
\text { NTHi, non-typeable Haemophilus influenzae; PCV-13, 13-valent } \\
\text { pneumococcal conjugate vaccine; PHiD-CV, 10-valent } \\
\text { pneumococcal non-typeable Haemophilus influenzae protein D } \\
\text { conjugate vaccine; QALY, quality-adjusted life-year; RCGP, Royal } \\
\text { College of General Practitioners. }\end{array}$} \\
\hline
\end{tabular}

limited due to disparities in country-specific epidemiology and healthcare systems. Finally, static models such as ours integrate net herd protection (ie, herd protection and type replacement combined) as a fixed effect at equilibrium. However, dynamic models may be more appropriate to capture these effects over time and in relation to population characteristics and vaccine coverage.

\section{CONCLUSIONS}

When considering the cost-effectiveness of a $2+1$ universal childhood vaccination programme in the UK from the perspective of medical costs, the strategy of using PHiD-CV dominates over use of PCV-13 when the vaccines are priced at parity. This result was primarily due to fewer AOM cases and associated cost-savings. Against the background of developing antibiotic resistance and reduced IPD incidence observed since the introduction of pneumococcal vaccination, our updated model suggests that deployment of the PHiD-CV vaccine would be of value in the UK. Finally, continuous active monitoring of epidemiological changes associated with pneumococcal vaccination programmes is important to inform future decision-making and healthcare policy in the UK.

Acknowledgements The authors would like to thank Jan Olbrecht (GSK Vaccines) for his support for the study and the publication, Fabien Debailleul (Business and Decision Life Sciences on behalf of GSK Vaccines) for publication coordination and Amrita Ostawal (freelancer on behalf of GSK vaccines) for medical writing assistance, and Carole Nadin (Fleetwith, on behalf of GSK Vaccines) for reviewing the manuscript for English usage.

Contributors ED provided substantial scientific input to the study and study report, critically reviewed the study report, and was involved in study and economic modelling development, populating models and determination of model settings, acquisition of data, determination of the model inputs and statistical data analysis. OL provided substantial scientific input to the study and study report, critically reviewed the study report, and was involved in method selection and development, economic modelling development, data mining and literature review, populating models and determination of model settings, acquisition of data, model inputs and assessment of robustness of results (sensitivity analysis). ET participated in the selection of model inputs and the acquisition of data. NVdV provided substantial scientific input to the study and study report, critically reviewed the study report, was involved in the method selection, development and determination of model settings, and participated in the development of the economic modelling and the sensitivity analysis. All authors provided intellectual contributions to this manuscript, critically reviewed the manuscript and have approved the final version.

Funding GlaxoSmithKline Biologicals SA funded this study and all costs associated with the development and the publishing of this present manuscript (internal GSK identifier: H0-12-8103).

Disclaimer Synflorix is a trade mark of the GSK group of companies.

Competing interests ED is an employee of the GSK group of companies and reports ownership of restricted shares from the GSK group of companies; OL reports that $(A)$ he was an external consultant and received payment on a contract basis from the GSK group of companies at the time of the study and (B) is married to a previous employee of the GSK group of companies owning restricted shares from the GSK group of companies; ET is an employee of the GSK group of companies; NVdV is an employee of the GSK group of companies and reports ownership of restricted shares from the GSK group of companies.

Provenance and peer review Not commissioned; externally peer reviewed.

Data sharing statement All data used in this study are presented in the manuscript and the supplementary files, or references to the original material are provided. Please contact the corresponding author shall you require any additional information. 
Open Access This is an Open Access article distributed in accordance with the Creative Commons Attribution Non Commercial (CC BY-NC 4.0) license, which permits others to distribute, remix, adapt, build upon this work noncommercially, and license their derivative works on different terms, provided the original work is properly cited and the use is non-commercial. See: http:// creativecommons.org/licenses/by-nc/4.0/

\section{REFERENCES}

1. Centers for Disease Control and Prevention. Measles. In: Atkinson W, Wolfe C, Hamborsky J, eds. Epidemiology and prevention of vaccine-preventable diseases. 12th edn. Washington DC: Public Health Foundation, 2012:173-92. Public Health Foundation [12th Ed], 173-192. 2011.

2. World Health Organisation. Immunisation, Vaccines and Biologicals. Estimated $\mathrm{Hib}$ and pneumococcal deaths for children under 5 years of age. 2008. http://www.who.int/immunization/monitoring surveillance/burden/estimates/Pneumo_hib/en/ (accessed 27 Aug 2014).

3. Health Protection Agency. General Information on Pneumococcal Disease. http://www.hpa.org.uk/web/HPAweb\&HPAwebStandard/ HPAweb_C/1203008864027 (accessed 23 Nov 2015).

4. Public Health England. https://www.gov.uk/government organisations/health-protection-agency (accessed 17 Sep 2014).

5. Elemraid MA, Sails AD, Thomas MF, et al. Pneumococcal diagnosis and serotypes in childhood community-acquired pneumonia. Diagn Microbiol Infect Dis 2013;76:129-32.

6. Falup-Pecurariu O. Lessons learnt after the introduction of the seven valent-pneumococcal conjugate vaccine toward broader spectrum conjugate vaccines. Biomed J 2012;35:450-6.

7. Ladhani SN, Slack MP, Andrews NJ, et al. Invasive pneumococca disease after routine pneumococcal conjugate vaccination in children, England and Wales. Emerging Infect Dis 2013;19:61-8.

8. Jokinen J, Palmu AA, Kilpi T. Acute otitis media replacement and recurrence in the Finnish otitis media vaccine trial. Clin Infect Dis 2012;55:1673-6.

9. National Health Services. The complete routine immunisation schedule 2013/14. https://www.gov.uk/government/uploads/system/ uploads/attachment_data/file/227651/8515_DoH_Complete_Imm_ schedule_A4_2013_09.pdf; (accessed 29 Aug 2014).

10. Prymula R, Peeters $P$, Chrobok V, et al. Pneumococcal capsular polysaccharides conjugated to protein $\mathrm{D}$ for prevention of acute otitis media caused by both Streptococcus pneumoniae and non-typable Haemophilus influenzae: a randomised double-blind efficacy study. Lancet 2006;367:740-8.

11. Domingues CM, Verani JR, Montenegro Renoiner EI, et al. Effectiveness of ten-valent pneumococcal conjugate vaccine against invasive pneumococcal disease in Brazil: a matched case-control study. Lancet Respir Med 2014;2:464-71.

12. Tregnaghi MW, Sáez-Llorens $X$, López $P$, et al. Efficacy of pneumococcal nontypable Haemophilus influenzae protein $D$ conjugate vaccine (PHiD-CV) in young Latin American children: a double-blind randomized controlled trial. PLoS Med 2014;11: e1001657.

13. Whitney CG, Pilishvili T, Farley MM, et al. Effectiveness of seven-valent pneumococcal conjugate vaccine against invasive pneumococcal disease: a matched case-control study. Lancet 2006;368:1495-502.

14. Deceuninck G, De Serres G, Boulianne N, et al. Effectiveness of three pneumococcal conjugate vaccines to prevent invasive pneumococcal disease in Quebec, Canada. Vaccine 2015;33:2684-9.

15. Jokinen J, Rinta-Kokko H, Siira $\mathrm{L}$, et al. Impact of ten-valent pneumococcal conjugate vaccination on invasive pneumococcal disease in Finnish children--a population-based study. PLOS ONE 2015;10:e0120290.

16. JCVI minutes Pneumococcal sub-committee meeting held on 30 May 2012. http://webarchive.nationalarchives.gov.uk/ 20120907090205/https://www.wp.dh.gov.uk/transparency/files/2012/ 07/JCVI-minutes-Pneumococcal-sub-committee-meeting-held-on-30May-2012.pdf (accessed 28 Aug 2014).

17. Andrews NJ, Waight PA, Burbidge $P$, et al. Serotype-specific effectiveness and correlates of protection for the 13-valent pneumococcal conjugate vaccine: a postlicensure indirect cohor study. Lancet Infect Dis 2014;14:839-46.

18. Synflorix. Summary of Product Characteristics. http://www.ema.europa eu/docs/en GB/document library/EPAR - Product Information/ human/000973/WC500054346.pdf (accessed 29 Aug 2014)

19. Prevenar-13. http://www.pfizer.co.jp/pfizer/company/press/2013/ documents/20131028_01.pdf (accessed 15 Aug 2014).
20. Dickson G. Acute otitis media. Prim Care 2014;41:11-18.

21. Wald ER. Acute otitis media and acute bacterial sinusitis. Clin Infect Dis 2011;52(Suppl 4):S277-83.

22. Cartwright K. Pneumococcal disease in western Europe: burden of disease, antibiotic resistance and management. Eur $J$ Pediatr 2002;161:188-95

23. Hung IF, Tantawichien T, Tsai YH, et al. Regional epidemiology of invasive pneumococcal disease in Asian adults: epidemiology, disease burden, serotype distribution, and antimicrobial resistance patterns and prevention. Int $J$ Infect Dis 2013;17:364-73.

24. Pelton SI, Huot H, Finkelstein JA, et al. Emergence of $19 \mathrm{~A}$ as virulent and multidrug resistant Pneumococcus in Massachusetts following universal immunization of infants with pneumococcal conjugate vaccine. Pediatr Infect Dis J 2007;26:468-72.

25. Van Eldere J, Slack MP, Ladhani S, et al. Non-typeable Haemophilus influenzae, an under-recognised pathogen. Lancet Infect Dis 2014;14:1281-92.

26. Knerer G, Ismaila A, Pearce D. Health and economic impact of PHiD-CV in Canada and the UK: a Markov modelling exercise. J Med Econ 2012;15:61-76.

27. Office for National Statistics, UK National Life Tables, 1980-82 to 2011-13. http://www.ons.gov.uk/ons/datasets-and-tables/index.html (accessed 11 Jun 2015).

28. Office for National Statistics. Population Estimates for UK, England and Wales, Scotland and Northern Ireland. http://www.ons.gov.uk/ ons/rel/pop-estimate/population-estimates-for-uk-england-andwales-scotland-and-northern-ireland/population-estimatestimeseries-1971-to-current-year/index.html (accessed 1 Apr 2015).

29. Hospital Episode Statistics (HES). The NHS Information Centre for Health and Social Care. Primary diagnosis: 4 character, 2013-14. http://www.hscic.gov.uk/catalogue/PUB16722/

hosp-outp-acti-2013-14-prim-diag-tab.xIsx (accessed 25 Sep 2015).

30. Pomeroy SL, Holmes SJ, Dodge PR, et al. Seizures and other neurologic sequelae of bacterial meningitis in children. $N$ Engl $\mathrm{J}$ Med 1990;323:1651-7.

31. Mclntyre PB, Berkey CS, King SM, et al. Dexamethasone as adjunctive therapy in bacterial meningitis. A meta-analysis of randomized clinical trials since 1988. JAMA 1997;278:925-31.

32. Kastenbauer S, Pfister HW. Pneumococcal meningitis in adults: spectrum of complications and prognostic factors in a series of 87 cases. Brain 2003;126:1015-25.

33. Auburtin M, Porcher R, Bruneel F, et al. Pneumococcal meningitis in the intensive care unit: prognostic factors of clinical outcome in a series of 80 cases. Am J Respir Crit Care Med 2002;165:713-17.

34. Johnson AP, Waight $\mathrm{P}$, Andrews $\mathrm{N}$, et al. Morbidity and mortality of pneumococcal meningitis and serotypes of causative strains prior to introduction of the 7-valent conjugant pneumococcal vaccine in England. J Infect 2007;55:394-9.

35. Melegaro A, Edmunds WJ. Cost-effectiveness analysis of pneumococcal conjugate vaccination in England and Wales. Vaccine 2004;22:4203-14.

36. Royal College of General Practitioners. Research \& Surveillance Centre. Weekly Returns Service Annual Report. 2011. http://www. rcgp.org.uk/clinical-and-research/research-and-surveillance-centre. aspx (accessed 17 Sep 2014).

37. Leibovitz E, Jacobs MR, Dagan R. Haemophilus influenzae: a significant pathogen in acute otitis media. Pediatr Infect Dis $J$ 2004;23:1142-52.

38. Hausdorff WP, Yothers G, Dagan R, et al. Multinational study of pneumococcal serotypes causing acute otitis media in children Pediatr Infect Dis J 2002;21:1008-16.

39. Hospital Episode Statistics (HES). The NHS Information Centre for Health and Social Care. Main procedures and interventions: 2013-14. http://www.hscic.gov.uk/catalogue/PUB16722/hosp-outp-acti-2013-14main-proc-inte-tab.xlsx (accessed 25 Sep 2015).

40. Williamson I, Benge S, Mullee M, et al. Consultations for middle ear disease, antibiotic prescribing and risk factors for reattendance: a case-linked cohort study. Br J Gen Pract 2006;56:170-5.

41. Health Care Supply Association. Health Service Cost Index. https:// nhsprocurement.org.uk/articles/health-service-cost-index (accessed 4 Dec 2015).

42. Curtis L. Unit costs of health and social care 2007, Personal Social Services Research Unit, University of Kent, Canterbury, 2007. http:// http://www.pssru.ac.uk/pdf/uc/uc2007/uc2007.pdf (accessed 28 Aug 2014).

43. Public Health England. Vaccine uptake guidance and the latest coverage data. https://www.gov.uk/government/statistics/cover-ofvaccination-evaluated-rapidly-cover-programme-2014-to-2015quarterly-data (accessed 11 Jun 2015).

44. Health Survey for England. 1996. http://www.archive.official-documents co.uk/document/doh/survey96/tab5-29.htm (accessed 5 Nov 2007). 
45. Bennett JE, Sumner WII, Downs SM, et al. Parents' utilities for outcomes of occult bacteremia. Arch Pediatr Adolesc Med 2000;154:43-8.

46. Cheng AK, Niparko JK. Cost-utility of the cochlear implant in adults: a meta-analysis. Arch Otolaryngol Head Neck Surg 1999;125:1214-18.

47. Oh $\mathrm{PI}$, Maerov $\mathrm{P}$, Pritchard $\mathrm{D}$, et al. A cost-utility analysis of second-line antibiotics in the treatment of acute otitis media in children. Clin Ther 1996;18:160-82.

48. Morrow A, De Wals P, Petit G, et al. The burden of pneumococcal disease in the Canadian population before routine use of the seven-valent pneumococcal conjugate vaccine. Can J Infect Dis Med Microbiol 2007;18:121-7.

49. Oostenbrink R, Oostenbrink JB, Moons KG, et al. Cost-utility analysis of patient care in children with meningeal signs. Int J Technol Assess Health Care 2002;18:485-96.

50. Chandran A, Herbert $H$, Misurski $D$, et al. Long-term sequelae of childhood bacterial meningitis: an underappreciated problem. Pediatr Infect Dis J 2011;30:3-6.

51. Waight PA, Andrews NJ, Ladhani SN, et al. Effect of the 13-valent pneumococcal conjugate vaccine on invasive pneumococcal disease in England and Wales 4 years after its introduction: an observational cohort study. Lancet Infect Dis 2015;15:535-43.

52. Miller E, Andrews NJ, Waight PA, et al. Effectiveness of the new serotypes in the 13-valent pneumococcal conjugate vaccine. Vaccine 2011;29:9127-31.

53. Heffron R. Pneumonia: with special reference to pneumococcus lobar pneumonia. Commonwealth Fund, 1939.

54. Kieninger DM, Kueper K, Steul K, et al. Safety, tolerability, and immunologic noninferiority of a 13-valent pneumococcal conjugate vaccine compared to a 7 -valent pneumococcal conjugate vaccine given with routine pediatric vaccinations in Germany. Vaccine 2010;28:4192-203.

55. Butler JC, Breiman RF, Campbell JF, et al. Pneumococcal polysaccharide vaccine efficacy. An evaluation of current recommendations. JAMA 1993;270:1826-31.

56. Singleton RJ, Butler JC, Bulkow LR, et al. Invasive pneumococcal disease epidemiology and effectiveness of 23-valent pneumococcal polysaccharide vaccine in Alaska native adults. Vaccine 2007;25:2288-95.

57. Vesikari T, Wysocki J, Chevallier B, et al. Immunogenicity of the 10-valent pneumococcal non-typeable Haemophilus influenzae protein D conjugate vaccine (PHiD-CV) compared to the licensed 7vCRM vaccine. Pediatr Infect Dis J 2009;28:S66-76.

58. Synflorix-Annex I-Summary of product characteristics (SmPC). http://www.ema.europa.eu/docs/en_GB/document_library/EPAR_Product_Information/human/000973/WC500054346.pdf (accessed 2 Dec 2015).

59. European Medicines Agency. Science Medicines Health. SynflorixProcedural steps taken and scientific information after the authoristation. http://www.ema.europa.eu/docs/en_GB/document library/EPAR_-_Procedural_steps_taken_and_scientific_information after authorisation/human/000973/WC500054350.pdf (accessed 2 Dec 2015).

60. Black SB, Shinefield HR, Ling S, et al. Effectiveness of heptavalent pneumococcal conjugate vaccine in children younger than five years of age for prevention of pneumonia. Pediatr Infect Dis $J$ 2002;21:810-5.

61. Hansen J, Black S, Shinefield $\mathrm{H}$, et al. Effectiveness of heptavalent pneumococcal conjugate vaccine in children younger than 5 years of age for prevention of pneumonia: updated analysis using World Health Organization standardized interpretation of chest radiographs. Pediatr Infect Dis J 2006;25:779-81.

62. Madhi SA, Kuwanda L, Cutland C, et al. The impact of a 9-valent pneumococcal conjugate vaccine on the public health burden of pneumonia in HIV-infected and -uninfected children. Clin Infect Dis 2005:40:1511-8.
63. Cutts FT, Zaman SM, Enwere G, et al. Efficacy of nine-valent pneumococcal conjugate vaccine against pneumonia and invasive pneumococcal disease in The Gambia: randomised, double-blind, placebo-controlled trial. Lancet 2005;365:1139-46.

64. Lucero MG, Nohynek H, Williams G, et al. Efficacy of an 11-valent pneumococcal conjugate vaccine against radiologically confirmed pneumonia among children less than 2 years of age in the Philippines: a randomized, double-blind, placebo-controlled trial. Pediatr Infect Dis J 2009;28:455-62.

65. Afonso ET, Minamisava R, Bierrenbach AL, et al. Effect of 10-valent pneumococcal vaccine on pneumonia among children, Brazil. Emerg Infect Dis 2013;19:589-97.

66. Kury C. Evaluation of the effectiveness of a 13-valent pneumococcal conjugated vaccine implemented in the municipality of Campos dos Goytacazes. Brazil: WSPID, 2013.

67. Eskola J, Kilpi T, Palmu A, et al. Efficacy of a pneumococcal conjugate vaccine against acute otitis media. $N$ Engl J Med 2001;344:403-9.

68. Fireman B, Black SB, Shinefield HR, et al. Impact of the pneumococcal conjugate vaccine on otitis media. Pediatr Infect Dis J 2003;22:10-16.

69. Palmu AA, Verho J, Jokinen J, et al. The seven-valent pneumococcal conjugate vaccine reduces tympanostomy tube placement in children. Pediatr Infect Dis J 2004;23:732-8.

70. Palmu AA, Jokinen J, Borys D, et al. Effectiveness of the ten-valent pneumococcal Haemophilus influenzae protein $D$ conjugate vaccine (PHiD-CV10) against invasive pneumococcal disease: a cluster randomised trial. Lancet 2013;381:214-22.

71. De Wals $\mathrm{P}$, Lefebvre B, Defay $\mathrm{F}$, et al. Invasive pneumococcal diseases in birth cohorts vaccinated with PCV-7 and/or PHiD-CV in the province of Quebec, Canada. Vaccine 2012;30:6416-20.

72. Pilishvili T, Lexau C, Farley MM, et al. Sustained reductions in invasive pneumococcal disease in the era of conjugate vaccine. $J$ Infect Dis 2010;201:32-41.

73. Whitney CG, Farley MM, Hadler J, et al. Decline in invasive pneumococcal disease after the introduction of protein-polysaccharide conjugate vaccine. $N$ Engl J Med 2003;348:1737-46.

74. Feikin DR, Kagucia EW, Loo JD, et al. Serotype-specific changes in invasive pneumococcal disease after pneumococcal conjugate vaccine introduction: a pooled analysis of multiple surveillance sites. PLoS Med 2013;10:e1001517.

75. Public Health England. Pneumococcal disease infections caused by serotypes in Prevenar 13 and not in Prevenar 7. https://www.gov.uk/ government/publications/pneumococcal-disease-caused-by-strainsin-prevenar-13-and-not-in-prevenar-7-vaccine/pneumococcaldisease-infections-caused-by-serotypes-in-prevenar-13-and-not-inprevenar-7 (accessed 23 Nov 2015).

76. National Institute for Health and Clinical Excellence. Discounting of health benefits in special circumstances. http://www.nice.org.uk/ media/955/4F/Clarification_to_section_5.6_of_the_Guide_to Methods_of_Technology_Appraisals.pdf (accessed 8 Apr 2014).

77. Office for National Statistics, 2011 census, age by single year. http:// www.ons.gov.uk/ons/search/index.html?newquery=QS103EW (accessed 28 Aug 2014).

78. Office for National Statistics, Statistical Bulletin, 2011 Annual Survey of Hours and Earnings (based on SOC 2010). http://www.ons.gov. uk/ons/dcp171778 256900.pdf (link to Excel file contained therein) (accessed 28 Aug 2014)

79. Department of Health. National tariff 2006/07: payment by results. 2007. http://webarchive.nationalarchives.gov.uk/20130107105354/ http://www.dh.gov.uk/en/Publicationsandstatistics/Publications/ PublicationsPolicyAndGuidance/DH_4127649 (accessed 1 Apr 2014).

80. Palmu AA, Jokinen $\mathrm{J}$, Nieminen $\mathrm{H}$, et al. Effect of pneumococcal Haemophilus influenzae protein D conjugate vaccine (PHiD-CV10) on outpatient antimicrobial purchases: a double-blind, cluster randomised phase 3-4 trial. Lancet Infect Dis 2014;14:205-12. 